\title{
STRUCTURAL, OPTICAL AND ELECTRICALCHARACTERIZATION OF POLYANILINE/ SILVER NANOCOMPOSITES
}

\author{
Jayasudha $S^{1}$, Priya $L^{2}$, K.T. Vasudevan ${ }^{3}$ \\ ${ }^{I}$ Department of Physics, CPGS, Jain University, Jayanagar 3rd Block, Bangalore-560011 \\ ${ }^{2}$ Department of Physics, LRG Government College for women, Tirupur- 641604 \\ svl.priya1@gmail.com \\ ${ }^{3}$ Department of PhysicsT. John College,86/1, Gottigere, Bannerghatta Road, Bangalore - 560083
}

\begin{abstract}
-
Nanocomposites of conducting polyaniline with Silver (PAni/Ag) have been synthesized by in-situ polymerization of aniline monomer using ammonium persulphate as an oxidizing agent. The weight percentage of silver varied from $3 \%$ to $25 \%$. The formation of PAni/Ag composites was assessed by X-Ray Diffraction (XRD), Field Emission Scanning Electron Microscope (FESEM) and UV-Vis spectroscopy. The broadening of sharp peaks in the XRD pattern indicates the presence of nano phase of Ag. The crystallite size of PAni/Ag nanocomposite from XRD is found to be $17.5 \mathrm{~nm}$. The FESEM image shows a nanoparticular structure of silver and it is also seen that the Ag nanoparticles are well dispersed in the polyaniline matrix. UV-Vis spectrogram of composites shows three peaks at $\sim 240 \mathrm{~nm}, 450 \mathrm{~nm}$ and $700 \mathrm{~nm}$ respectively in addition to $\sim 198$ and $\sim 324 \mathrm{~nm}$ peak of Pure PAni. The peak around $450 \mathrm{~nm}$ confirms the presence of radical cation or polaron transition. The peak around $700 \mathrm{~nm}$ is the characteristic peak of PAni Emeraldine base. The peak around $240 \mathrm{~nm}$ could be assigned to the characteristic absorption of small metallic Ag cluster. The graph of hvVs (ahv)2 was also plotted. The PL spectrum of PAni/Ag was excited with an excitation wavelength of $320 \mathrm{~nm}$. Two peaks are observed at a wavelength of $\sim 370 \mathrm{~nm}$ and $600 \mathrm{~nm}$. The DC conductivity of Pure PAni and its composites have been measured in the temperature range from $25^{\circ} \mathrm{C}$ to $150{ }^{\circ} \mathrm{C}$. The DC electrical study of PAni/Ag nanocomposite clearly indicates that the Ag nanoparticles increase the electrical conductivity of polyaniline nanocomposites as compared to polyaniline.
\end{abstract}

Key words: XRD, Photoluminescence, Polyaniline, Differential scanning calorimetry

\section{INTRODUCTION}

During the last decade there has been a wide spread interest in conducting polymers. Polyaniline (PAni) is one of the most useful conducting polymers due to its high degree of stability [1]. It is one of the useful conducting polymers due to its ease of synthesis and simplicity in preparation [2, 3].

Conducting polymer has the electronic properties of semiconductors and at the same time it has got the mechanical properties of the polymer [2]. Due to global warming and the exhaustion of other fossil based fuels alternate renewable energies obtained by solar cells have attracted much attention. Thus the synthesis of novel conducting polymers and study of their physical properties has been of prime importance. There are many unresolved problem concerning the structure and properties of PAni because of the complexities in the molecular structure [4].

The conducting polymers are characterized by conjugated alternate single and double bonds. This feature originates from $\pi$-electron. Conducting polymers helped in understanding the fundamental chemistry and physics of $\pi$ bonded macromolecules. The $\pi$ electron of conjugated polymers shows wide range of features in electrical and optical properties. [5]. The charge carriers in polyaniline are generally considered as polaron and bipolaron. The physical properties of polyaniline depend on the choice of the counter ion called dopants [6]. The approach of chemical synthesis and the functional dopants in the polymer matrix eliminates the use of volatile dopants.

Generally PAni exists in four different forms (leucoemarldine base, pernigraniline base, emarldine base and emarldine salt (ES)). Among the different form of PAni only ES is electrically conducting and can be synthesized electrochemically or chemically in an acidic medium by means of oxidants [7]. The chemical oxidation polymerization is practically viable due to feasibility of synthesis mechanism for producing large scale PAni powder.

The inception of Nitrogen heteroatom in between Phenyl rings of PAni backbone chain results in the significant properties of PAni. The formation of polarons and bipolarons in the energy band led to the possibility of different types of charge conduction. Oxidation and protonation of amine nitrogen atoms causes the change in the electrical and optical properties of PAni [8].

The metal nanoparticles are prepared due to its unique physical and chemical properties. Stable silver nanoparticles are widely used as photosensitive components, photo catalysts and chemical analysis [9].Silver nanoparticles also 
possess many properties like electrical conductivity; antimicrobial activity etc., the incorporation of metal nanoparticles could effectively improve the electrical, optical properties of polyaniline composites. The electrical conductivity of such composites might also depend upon the molecular structure of polymer matrix [10]. To achieve nanoparticle dispersion is difficult because of high surface area to volume ratio.

In the present study, PAni/Ag nanocomposites with different Ag concentration were synthesized by in situ polymerization techniques. In this current work the structural, morphological, optical and electrical characterizations of nanocomposites are reported.

\section{EXPERIMENTAL TECHNIQUE}

Analytical grade Aniline monomer, Hydrochloric acid and Ammonium persulphate was purchased from Qualigens, and silver nitrate is purchased from Merck chemicals.

\subsection{Synthesis of Pure Pani}

The chemical polymerization of Aniline was carried out in the presence of hydrochloric acid. The prepared $\mathrm{HCl}$ solution is divided into two beakers. Appropriate amount of aniline solution is mixed in $3 \mathrm{M} \mathrm{HCl}$ solution in first beaker and $2 \mathrm{M}$ of Ammonium per sulphate is added to $3 \mathrm{M} \mathrm{HCl}$ in the second beaker. Then Ammonium per sulphate solution was added drop by drop to the prepared aniline solution which is maintained around 2 to $4^{\circ} \mathrm{C}$ till the colour changes to blue and then finally to dark green colour. Dark green colour indicates the formation of polyaniline. This solution is kept for stirring overnight to polymerize completely. Polymerized sample was purified by dialyzing against distilled water and the final solution is kept for drying at room temperature. Then the synthesised PAni is finally crushed to obtain a fine powder

\subsection{Synthesis of Pani/Ag Nanocomposites}

$0.05 \mathrm{M} \mathrm{AgNO}$ solution is prepared. Then the prepared $\mathrm{AgNO}_{3}$ solution is added drop by drop to the prepared polyaniline solution. It is allowed for continuous stirring and the prepared solution is dialysed against distilled water. The final solution is purified as before and kept for drying.The samples of different compositions containing 0.01, 0.03 and0.1 $\mathrm{M} \mathrm{AgNO}_{3}$ are also prepared by the same method.

\section{CHARACTERIZATION TECHNIQUES}

Structural characterization of the prepared PAni and PAni/Ag composites were carried out in Brukuer X-ray diffractrometer using $\mathrm{Cu}-\mathrm{K} \alpha$ radiation. The morphology of the sample was observed using FESEM in NEON 40 cross beam Carl Zeiss instrument. UV - Visible spectroscopy analysis was done using the Schimadzu 1800 UV - Vis spectrophotometer sweeping the incident wavelengths between $190 \mathrm{~nm}$ to $1200 \mathrm{~nm}$. Photoluminescence study was carried out in a JY Fluorolog -3-11 Spectroflurometer between the wavelength of 300 and $700 \mathrm{~nm}$. DSC analysis is done in the temperature range of 0 to $350^{\circ} \mathrm{C}$ using Mettler Toledo DSC1 instrument.

The polymer nanocomposites were ground in a mortar pestle to obtain fine powder form. The powder is pressed to form pellets of $1 \mathrm{~cm}$ in diameter and thickness varies 1 to $3 \mathrm{~mm}$ by applying pressure of $70 \mathrm{MPa}$ in a hydraulic press. The prepared pellets are annealed for 2 hours. The mild annealing that the samples might receive will help the PAni to experience more ordering. The same sample was used to measure the electric conductivity $(\sigma)$ using two probe technique from a temperature range of $25^{\circ} \mathrm{C}$ to $150^{\circ} \mathrm{C}$.

\section{RESULTS AND DISCUSSION}

\subsection{Xrd Studies}

Fig. 1 shows XRD pattern of pure PAni and PAni silver nanocomposites. Pure PAni shows two peaks of $2 \theta$ at $\sim 20^{\circ}$ and $25^{\circ}$ which correspond to (100) and (110) crystal planes $[11,12]$. In addition to PAni Peaks, peaks corresponding to silver were also found at $\sim 32^{\circ}(101), \sim 45^{\circ}(200), \sim 65^{\circ}$ (220) and $\sim 78^{\circ}$ (311) plane [13]. All the reflections correspond to pure silver metal with face centred cubic symmetry. [14].

Degree of crystallinity of PAni/Ag composites were also calculated by X-ray diffraction analysis. The values were found to vary from $20 \%$ to $73 \%$. Degree of crystallinity increases with increase in concentration of nano silver clearly indicating the homogeneous distribution of nanoparticles in the polymer matrix. The particle size of the crystalline particle can be determined using Debye Scherrer formula and it is found that the grain size of PAni/Ag nanocomposite is $(\sim 17.5 \mathrm{~nm})$.

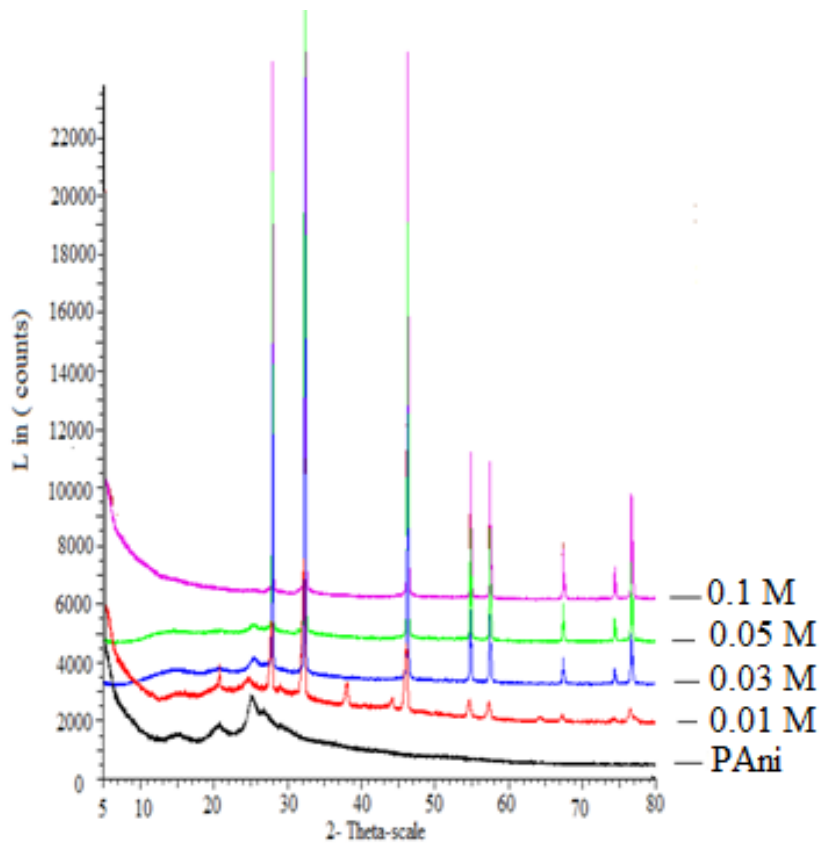

Fig: 1 XRD spectrum of PAni and PAni/Ag nanocomposites

\subsection{Morphological Study}

Fig. $2 \mathrm{a}$ and $2 \mathrm{~b}$ shows FESEM images of PAni and PAni/Ag nanocomposites, respectively. 
FESEM shows that the samples of pure PAni have a fibrillar structure. PAni chains are prepared in acidic media and exist in the form of poly cations [15]. In PAni/Ag nanocomposites, silver nanoparticles are well adhered on the PAni matrix, due to strong affinity of silver to nitrogen of PAni and it is also observed that Ag has a strong effect on the PAni's morphology; it shows a transformation in morphology of PAni particles from pure PAni to its nanocomposites. The formation of a relatively large cluster with Ag dispersion could be attributed to silver migration and aggregation. As the percentage of silver increases there is a change in morphology of PAni which helps in transportation through carbon backbone of PAni.

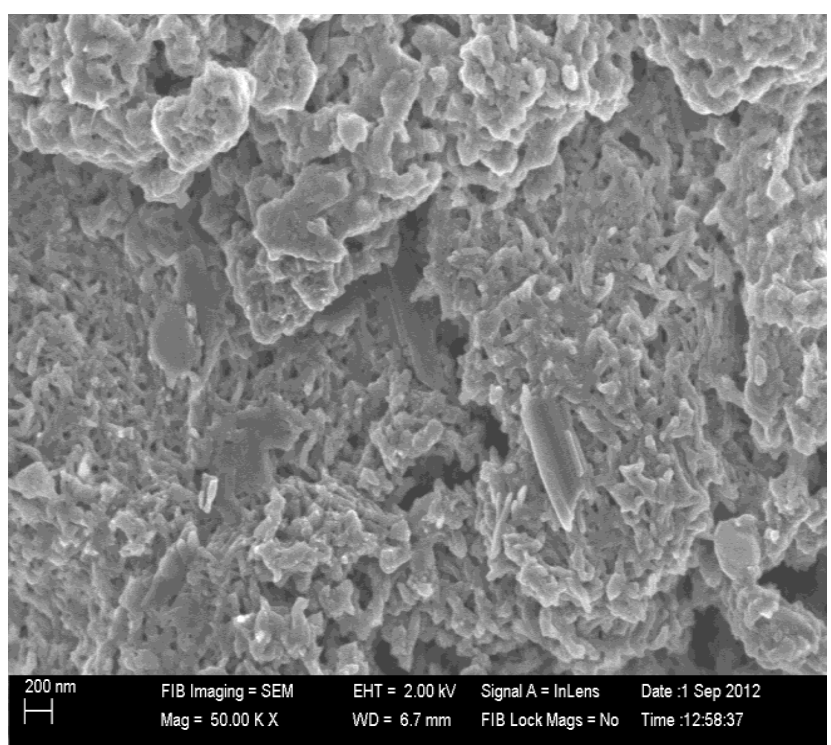

Fig. 2. a. FESEM image of pure PANI

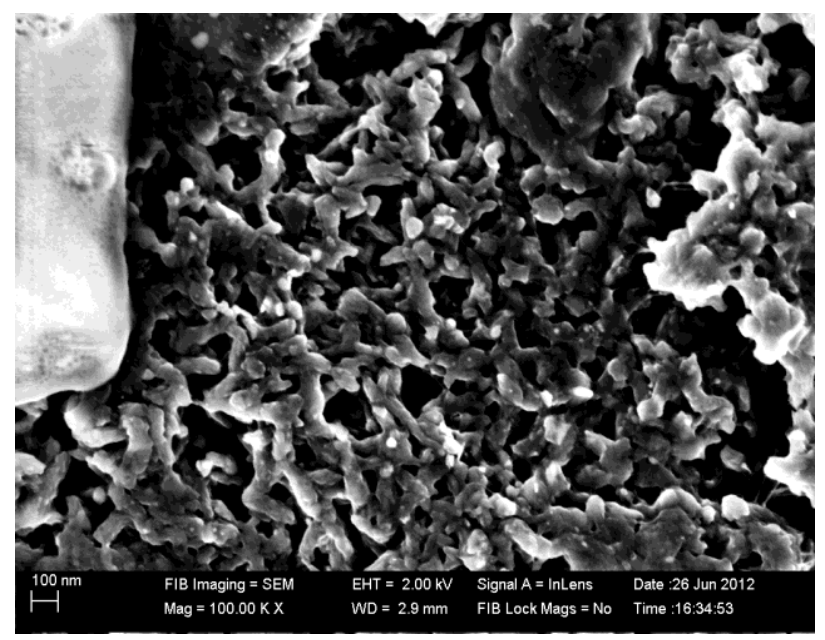

Fig 2.b. FESEM image of PANI/Ag nanocomposites

\subsection{UV - Vis Study}

Fig. 3 represents the UV-Vis graph of Pure PAni and its composites. UV-Vis spectrogram of the nanocomposites shows three peaks at $\sim 240 \mathrm{~nm}, 420 \mathrm{~nm}$ and a broad peak around $580 \mathrm{~nm}$ to $850 \mathrm{~nm}$ respectively in addition to $\sim 198$ and $\sim 324 \mathrm{~nm}$ peak of Pure PAni. The peak at $324 \mathrm{~nm}$ is due to $\pi-\pi^{*}$ transition of benzenoid ring of PAni, which is related to the extent of conjugation bond between the adjacent phenylene ring in the polymeric chain. The absorption band around $420 \mathrm{~nm}$ is attributed to the doping level of PAni which is predominant in lower percentage and the same is not significant at higher concentration. The peak also confirms the presence of radical cation or polaron transition. As the peak of PAni and the peak of silver gets superimposed at this position in the composites this peak can also be assigned to surface plasmon resonance absorption of the electrons in the conducting silver bands which indicates that the nanoparticles are present in the nanocomposites [16].

The intensity of polaron absorption band is related to the doping of PAni. Generally the absorption of polaron band decreases for PAni with higher doping level as the radical cation is distributed uniformly [17]. The broad peak in the range 580 to $850 \mathrm{~nm}$ might be due to the excitation like transition in quinoid units corresponding to the semiconducting phase of PAni [18]. This peak might be due to intermediate species, possibly a mixture containing major amount of conducting emeraldine salt. The peak around $\sim 240 \mathrm{~nm}$ could be assigned to the characteristic absorption of small metallic Ag cluster.

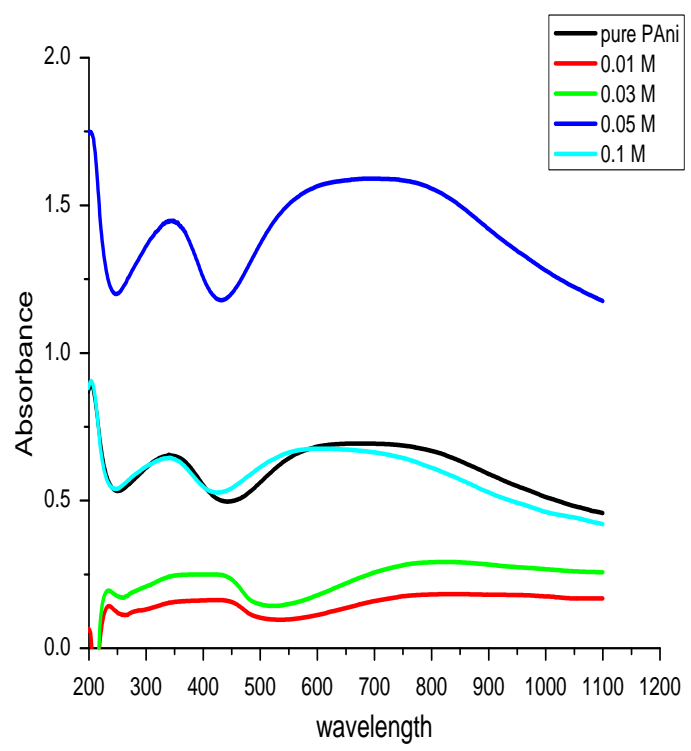

Fig.3 UV-Vis study of PAni and PAni/Ag nanocomposites

\subsection{Photoluminescence Analysis}

Fig. 4 shows PL spectrum of PAni/Ag was excited with an excitation wavelength of $320 \mathrm{~nm}$. The excitation wavelength is taken as $320 \mathrm{~nm}$ to excite silver nanoparticle may be due to $\pi^{*}$ transition of the benzenoid unit of polyaniline. Two peaks are observed at a wavelength of $\sim 370 \mathrm{~nm}$ and $600 \mathrm{~nm}$ in composites. Pure PAni reveals three peaks at $\sim 370^{\circ}, 580^{\circ}$ and $610^{\circ}$. The peak of PAni and composites at $\sim 370 \mathrm{~nm}$ attributed to the benzenoid groups causing the emission in polymers [19] and the peak $\sim 610 \mathrm{~nm}$ is due fully protonated species, whereas, the composite peak at $\sim 600 \mathrm{~nm}$ is the indication for the preceding cluster growth process and the appearance of nanoparticles. 


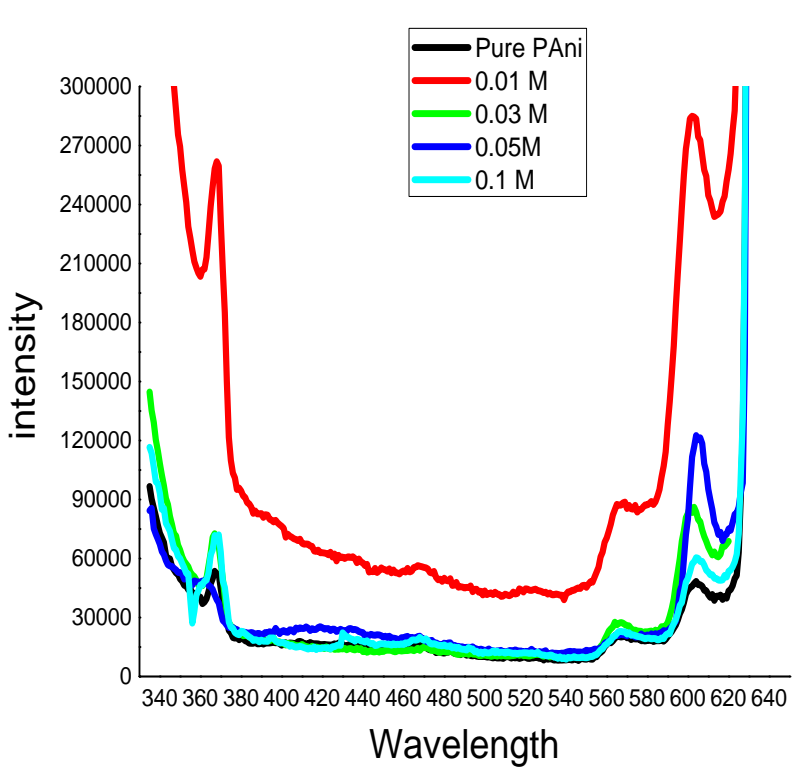

Fig.4 Photoluminescence of Pure PAni and PAni/Ag nanocomposites

\subsection{Conductivity}

To understand the DC conductivity effect of Ag nanoparticles on PAni, a comparison was made between pure PAni and its composites. Fig. 6 shows the temperature dependence of DC conductivity in the range $25^{\circ} \mathrm{C}$ to $150^{\circ} \mathrm{C}$. From this figure it is evident that as the concentration of silver increases conductivity increases. As the concentration of silver is increased in the composites, minimum increase of 2 orders of magnitude and maximum of 6 orders of magnitude are inferred in composites compared to PAni. This increase in conductivity after doping may be due to increase in the number of polarons formed during the doping process. The electrical conductivity of conducting polymers results from mobile charge carriers that are introduced through doping.

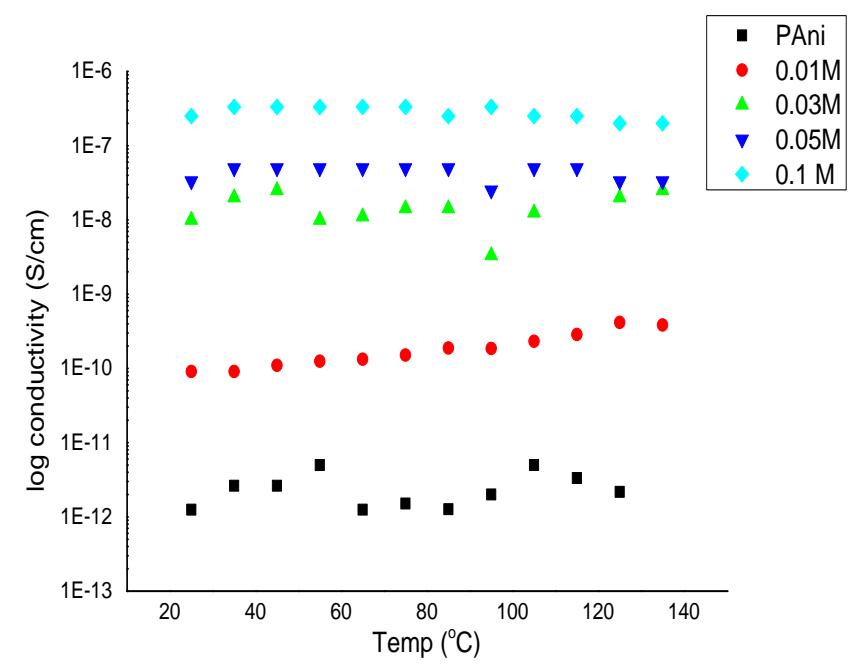

Fig.5 DC conductivity of Pure PAni and PAni/Ag nanocomposites

\section{CONCLUSION}

PAni/Ag nanocomposites were successfully synthesized by in-situ polymerization at different $\mathrm{Ag}$ concentrations. The formations of $\mathrm{Ag}$ nanoparticles in the nanocomposites were confirmed by XRD, FESEM and UV-Vis spectroscopy. The optical characteristic was confirmed by photoluminescence studies. The samples are thermally characterized by DSC. The electrical study was confirmed by DC conductivity. The fibrillar structure of PAni and the linkage of these PAni chains with silver nanoparticles incorporated in it could be the reason for the better conductivity in the PAni nanocomposites. The XRD patterns shows that as the concentration increases the intensity increases. It also indicated that the crystalline phase of Ag. FESEM analysis showed the uniform dispersion of $\mathrm{Ag}$ nanoparticles in the PAni matrix. UV-Vis analysis shows both the PAni and Ag peaks, and Photoluminescence analysis shows that composites shows a similar shape like PAni, indicates that it is an efficient way to modify the intensities. DC electrical conductivity of polyaniline/Ag nanocomposites was found to increase when compared to pure polyaniline. The DC conductivity of the samples was found to increase with temperature which is indicative of the metallic nature of the polymer composites. The activation energy of the samples is also calculated.

\section{REFERENCES}

[1]. Kerileng M. Molapo et al, "Electronics of conjugated polymers (I): Polyaniline,” Int. J. Electrochem. Sci., 7, 2012, 11859 - 11875 .

[2]. YomenAtassi, Mohammad Tally and Mazen Ismail, "Synthesis and characterization of chloride doped polyaniline by bulk oxidative chemical polymerization doping effects on electrical conductivity," Higher Inst. for Appl. Sci. and Technol, HIAST, P.O. Box 31983, Damascus, Syria.(2008)

[3]. J. Vivekanandan, V. Ponnusamy, A. Mahudeswaran and P. S. Vijayanand, "Synthesis, characterization and conductivity study of polyaniline prepared by chemical oxidative and electrochemical methods," Arch. of Appl. Sci. Res., 3(6), 2011, 147 - 153.

[4]. H.G. Taleghani, M. Aleahmad and H. Eisazadeh, "Preparation and Characterization of polyaniline Nanoparticles using various solutions," World Appl. Sci. J., 6(12), 2009, 1607 - 1611.

[5]. B. Kavitha, K. Sivakumar and N Narsimlu, "synthesis and characterization of polyaniline nano-fibres," Indian J. Pure and Appl.Phys, 51, 2013, 207 - 209.

[6]. Sharma I D et al., "Structural, Optical, Morphological and Electrical characteristics of Polyaniline for device applications," Indian J. Eng.\&Mater.sci., 20, 2013, 145- 149.

[7]. Ersel Ozkazanc, SibelZor, Hatice Ozkazanc, H. YukselGuney and Ufuk Abaci, "Synthesis, characterization and dielectric behaviour of (ES)-form polyaniline/cerium (III)-nitrate-hexahydrate composites," Mater. Chem. and Phys, 133, 2012, 356 362 . 
[8]. Mohammed Ahmed Ali et al, "Synthesis of conducting polyaniline nanocomposties by radiation doping," J. Eng. Sci. and Technol., 2, 2007, $111-118$.

[9]. Mansor Bin Ahmad, Kamyar Shameli, Majid Darroudi, Wan MdZin Wan Yunus and Nor Azowa Ibrahim, "Synthesis and characterization of silver/clay nanocomposites by chemical reduction method," Am. J. Applied Sciences 6(11):2009, 1909 - 1914.

[10].Safenaz M.Reda, and Sheikha M. Al-Ghannam, "Electrical properties of polyanilne composites with silver nanoparticles," Adv. in Materi. Phys. and Chem, 2, 2012, $75-81$.

[11].Pouget J P, Jozefowicz ME, Epstein AJ, Tang X and MacDiarmid," $X$ - ray structure of Polyaniline,"Macromol, 24, 1991, 779-789.

[12].Krishna B M et al, "Electrical properties of polyaniline doped with metal ions," J. phys. D: 42, 2009, 095404.

[13].K. Gupta, P.C. Jana and A.K. Meikap, "Optical and Electrical transport properties of polyaniline-silver nanocomposites," Synth. Metal, 160, 2010, 1566 1573.

[14].T. Theivasanthi and M. Alagar, "Electrolytic synthesis and characterization of silver nanopowder," Nano Biomed Eng, 4(2),2011, 58 - 65.

[15].SajjadSedaghat and FaribaGolbaz, "In situ oxidative polymerization of aniline in the presence of manganese dioxide and preparation of polyaniline $/ \mathrm{MnO}_{2}$ nanocomposite," J.Nanostruct.in Chem., 3, 2013, 1-3.

[16].Arup choudhury, "Polyaniline/silver nanocomposites: Dielectric properties and ethanol vapour sensitivity," Sensors and Actuators B, 138, 2009, 318 - 325.

[17].P.S. Khiew, N.M. Huang, S. Radiman and Md. Soot Ahmad, "Synthesis and characterization of conducting polyaniline - coated cadmium sulphide nanocomposites in reverse microemulsion, Mater.Lett" 58, 2004, $516-521$.

[18].Guicun li., Chuanqinzhang.,Hongrui Peng., Kezhengchen. and zhikun Zhang., Hollow self-Doped Polyaniline Micro/Nanostructures: Microspheres, Aligned Pearls and Nanotubes, Macromol Rapid Commun., 29, 2008,1954 - 1958

[19].Sipho E. Mavundla, Gerald F. Malgas, David E. Motaung and Emmanuel. I. Iwuoha, "Physiochemical and morphological properties of Poly (aniline-copyrrole)," J. Mater. Sci., 45, 2010, 3325 - 3330. 